Revue des patrimoines

36 | 2018

Les archives photographiques de presse, pratiques comparées et enjeux méthodologiques

\title{
La propriété matérielle des archives photographiques de presse. Enjeux juridiques
}

On some legal issues involved in the copyright ownership of press photo archives

\section{Samuel Bonnaud-Le Roux}

\section{(2) OpenEdition}

Journals

Édition électronique

URL : http://journals.openedition.org/insitu/17906

DOI : 10.4000/insitu. 17906

ISSN : 1630-7305

Éditeur

Ministère de la culture

Référence électronique

Samuel Bonnaud-Le Roux, «La propriété matérielle des archives photographiques de presse. Enjeux juridiques », In Situ [En ligne], 36 | 2018, mis en ligne le 15 octobre 2018, consulté le 02 mai 2019. URL : http://journals.openedition.org/insitu/17906 ; DOI : 10.4000/insitu.17906

Ce document a été généré automatiquement le 2 mai 2019.

\section{cc)}

In Situ Revues des patrimoines est mis à disposition selon les termes de la licence Creative Commons Attribution - Pas d'Utilisation Commerciale - Pas de Modification 4.0 International. 


\title{
La propriété matérielle des archives photographiques de presse. Enjeux juridiques
}

On some legal issues involved in the copyright ownership of press photo archives

\author{
Samuel Bonnaud-Le Roux
}

\section{Introduction}

De nombreux services d'archives, musées ou bibliothèques conservent des fonds photographiques de presse. Régulièrement, face à ces fonds souvent volumineux, hétéroclites, composés de photographies aux provenances très diverses et aux objets non authentifiés, ils peinent à déterminer la propriété matérielle des différents éléments composant ces fonds.

Il est possible d'identifier trois catégories de producteurs d'archives photographiques de presse :

- Un photoreporter ou photojournaliste. Ainsi en est-il par exemple du fonds Jean Lattes, riche de plusieurs dizaines de milliers de phototypes, conservé par les archives départementales des Yvelines ${ }^{1}$. A priori, le producteur du fonds confié étant l'auteur des clichés, on peut légitimement admettre que la question de la propriété des supports matériels est réglée.

- Une agence de presse.

- Un journal ou un groupe de presse désireux de préserver sa photothèque en en confiant la conservation à un service d'archives. C'est le cas du fonds de La Montagne aux archives départementales du Cantal et de Corrèze ${ }^{2}$.

Quel que soit le producteur, des incertitudes juridiques entourent la propriété des fonds : l'entité ayant effectué le versement auprès du service d'archives en était-elle vraiment propriétaire? Retracer le parcours de chaque négatif, diapositive ou tirage argentique pris individuellement s'avère particulièrement complexe en raison de la diversité des 
pratiques des acteurs du photojournalisme, qui ne font pas l'objet d'une normalisation particulière.

L'accueil d'un fonds de photographies est rarement assorti d'archives papier ou d'informations relatives à la production des dites photographies. Dans le cas de photographies de presse, qui relève d'un usage bien particulier de la photographie qui lie commanditaires de reportages, photographes, diffuseurs et utilisateurs, l'absence de connaissance des relations entre les différents acteurs du processus de production et de diffusion complexifie la tâche du service d'archives, dernier maillon de la chaîne.

L'histoire et la trajectoire des photographies de presse sont rarement linéaires, ce que révèlent de nombreux conflits portant sur cette question. Produites à un moment donné, elles ont pu passer de main en main sans qu'un contrat n'ait prévu à chaque étape un transfert de propriété. Cette difficulté de traçabilité peut être amplifiée si l'agence de presse, exploitant initial d'un cliché, a disparu à la suite d'une faillite, par exemple, ou a été absorbée, sans que la question de la propriété des supports photographiques n'ait été tranchée. Il importe donc d'examiner plus en détail les relations entre intervenants du secteur.

Lorsqu'un éditeur de presse utilise un cliché pour illustrer ses articles sur l'actualité, les photographies qu'il commande pour l'occasion ont trois sources principales: des photographes salariés de l'éditeur lui-même, des photographes pigistes ou bien des agences spécialisées. Dans chaque cas de figure, le lien juridique noué entre les partenaires autorise, sur le fondement du droit d'auteur, le titre de presse à reproduire les photographies en contrepartie d'une rémunération, mais ne se prononce pas forcément sur le sort des supports eux-mêmes. Ces dernières années, des conflits ont surgi opposant des agences de presse aux photographes, qui portent sur la restitution aux photographes de leurs originaux ou l'accès à ces derniers lorsque les photographes n'ont pas conservé de copies en amont.

La jurisprudence relative aux pratiques de transfert de propriété des supports photographiques dans les relations entre photographes et agences de presse retiendra d'abord notre attention, pour comprendre le contexte plus général des fonds conservés par des services d'archives. Puis, un examen des différentes modalités d'entrée des archives de presse dans les collections publiques permettra de saisir leur impact sur la propriété matérielle des archives photographiques. Enfin, sera abordée la possibilité pour des services d'archives d'appliquer la règle de la prescription acquisitive aux fonds photographiques privés dont les modalités d'entrée dans les collections sont inconnues, en l'absence de document précisant l'intention du producteur du fonds.

\section{La chaîne de propriété des supports photographiques dans le secteur de la presse}

Un cliché pris par un photographe de presse constitue une « œuvre de l'esprit » protégée par le droit d'auteur, s'il constitue une création de forme originale, c'est-à-dire s'il exprime des choix libres et créatifs de la part de son auteur. Plus précisément, la protection du droit d'auteur porte sur l'œuvre elle-même, et non sur les différents supports sur lesquels elle est fixée - négatifs originaux, plaques de projection, autochromes, diapositives, tirages argentiques, fichiers numériques. Cette distinction entre le droit de propriété incorporelle protégéant une œuvre et le droit de propriété 
matérielle portant sur le support dans lequel cette œuvre est incorporée est un des principes fondateurs du droit d'auteur, affirmé à l'article L. 111-3 du Code de la proprité intellectuelle. Il en découle que deux régimes juridiques différents s'appliquent à ces deux objets : le Code civil pour le support et le Code de la propriété intellectuelle pour l'œuvre. Des conflits peuvent apparaître lorsque les personnes détentrices du support et des droits d'auteur ne sont pas les mêmes.

Par exemple, un musée propriétaire des œuvres d'art qu'il expose n'en détient pas ipso facto les droits de reproduction qui s'y attachent. La Cour de cassation a ainsi jugé en 1966, au sujet d'une sculpture de Brancusi, que le transfert du droit de propriété d'une œuvre d'art, qu'il intervienne à titre onéreux ou gratuit, n'entraîne pas, à moins de convention contraire, la cession du droit de reproduction. En l'espèce, le legs particulier de ses œuvres consenti par le sculpteur à un tiers ne privait pas ses légataires universels du droit de reproduction ${ }^{3}$.

En matière de photographie, il est parfois délicat de dissocier l'œuvre du support dans lequel elle s'inscrit. La distinction entre propriété matérielle et propriété intellectuelle a pour conséquence que lorsqu'un photographe cède ses droits d'auteur sur un cliché à une agence de presse tout en lui remettant un exemplaire original pour qu'elle puisse en réaliser des tirages ultérieurs, il en reste propriétaire si le contrat ne précise rien quant à la propriété matérielle des supports. La cour d'appel de Paris, dans une décision du 26 mars 1992, a ainsi estimé que le photographe qui cède le droit de reproduction sur ses photographies pour une utilisation déterminée conserve, sauf convention expresse, la propriété matérielle de ses clichés.

Les pratiques varient selon les acteurs. Certaines agences proposent un contrat explicite de dépôt des clichés dans leurs locaux, à charge de les restituer ensuite au photographe, mais d'autres proposent des contrats ne prévoyant pas toujours de dispositions particulières sur les tirages : sont-ils seulement mis à disposition temporairement, ou cédés? Que se passe-t-il dans le silence du contrat? Il faut alors observer au cas par cas quelle a été l'intention des parties. La cour d'appel de Versailles a jugé par exemple, dans une décision du 24 janvier 2002, que la cession du droit de reproduction de clichés emporte celle de leur propriété matérielle lorsque le photographe a facturé distinctement les frais de reportage et les frais techniques.

En pratique, avec l'explosion du nombre de sources d'images sont apparus ces dernières années des conflits opposant des photographes à leur agence, portant la plupart du temps sur la restitution aux photographes de leurs originaux ou l'accès à ces derniers si le photographe n'a pas conservé de copies de ces derniers au préalable.

\section{Les relations entre agences de presse et photographes : le sort des tirages}

De même que les autres secteurs de la création, la photographie a été profondément touchée par la révolution numérique enclenchée depuis le début des années 1990, qui a bouleversé tant le nombre de canaux de diffusion des images de toute nature que les relations entre les intervenants traditionnels. L'adaptation à cette nouvelle donne conduit les agences de presse à numériser les anciens supports photographiques qu'elles détiennent. Le lancement de vastes projets de numérisation a placé au premier plan les questions d'identification du propriétaire matériel de chaque cliché pris individuellement au sein d'un fonds. 
Lorsqu'elles fournissent à leurs clients des clichés pris par des photographes indépendants, les agences photographiques de presse ${ }^{4}$ 'en détiennent pas forcément les droits d'auteur et peuvent n'être que dépositaires des clichés. Dans ce cas, un contrat de mandat unit le photographe et l'agence, qui autorise cette dernière à délivrer aux utilisateurs les autorisations d'exploitation des photographies. Ce mandat implique juridiquement un usage commercial des photographies mais aucun transfert de propriété, ni matérielle ni incorporelle. En contrepartie, l'agence reverse au photographe une partie des sommes qu'elle perçoit des utilisateurs. En l'absence de clause de cession de droits, l'agence ne peut revendiquer aucun droit sur les œuvres d'un photographe, que celui-ci d'ailleurs soit salarié de l'agence ou contributeur extérieur.

Un jugement du tribunal de grande instance de Paris du 21 décembre 2007 a ainsi considéré que la remise de photographies à une agence aux fins d'exploitation est constitutive d'un contrat de dépôt qui met à la charge du dépositaire une obligation de restitution ${ }^{5}$. Dans cette affaire, une agence de presse photographique avait perdu une grande partie des clichés et souhaitait requalifier le contrat de dépôt en un contrat de coproduction, ce qui aurait permis de mettre à la charge du photographe la part des risques liés à d'éventuelles pertes. Le tribunal a estimé au contraire que le rôle de l'agence était « exclusif de toute co-production» dans la mesure où elle n'intervenait pas «dans la réalisation même des photographies » et n'en contrôlait pas «la conception». Ainsi, le dépositaire était tenu d'une obligation de restitution des clichés dont seule la force majeure pouvait l'exonérer. Cette solution a été rappelée depuis lors dans des décisions ultérieures, notamment un jugement du tribunal de grande instance de Nanterre du 22 janvier 2008.

Les difficultés se cristallisent autour de contrats, parfois anciens, passés entre les agences et les photographes, qui n'ont pas forcément été adaptés pour prendre expressément en compte la conversion au format numérique de tirages analogiques et les nouvelles exploitations liées au Web. Dans une décision de 2012, la Cour de cassation avait censuré l'arrêt d'une cour d'appel pour ne pas avoir recherché, comme elle y était invitée, si les numérisations et mises en ligne de photographies par une agence de presse n'étaient pas impliquées, en l'absence de clause contraire, par le mandat reçu de commercialiser ces images et le besoin d'en permettre la visualisation par des acheteurs potentiels ${ }^{6}$.

Il s'agissait en l'espèce d'un photographe qui, au terme de son contrat de travail avec une agence de presse, avait conclu un accord selon lequel cette dernière pourrait continuer d'exploiter les archives photographiques moyennant le versement à l'auteur d'une redevance. Le photographe assigna quelques années plus tard l'agence en raison de la perte de tirages originaux et de la numérisation et de la diffusion sur le site Web de l'agence d'un grand nombre de ses photographies sans son autorisation explicite. L'agence avançait en réponse que son activité consistait dans la commercialisation par tous moyens de photographies dont elle disposait dans ses archives, et qu'une exploitation sur Internet relevait de l'exploitation normale des archives photographiques et des usages du secteur. La cour d'appel avait relevé que ni le contrat de travail et son avenant ni le protocole ne contenaient de disposition concernant ce type d'exploitation, et avait donc condamné l'agence pour contrefaçon sur le fondement des articles L. 122-4 et L. 131-3 du Code de la propriété intellectuelle.

La cassation de la décision de la cour d'appel en ce qu'elle avait condamné l'agence pour contrefaçon avait été critiquée à l'époque, car la Cour de cassation semblait ouvrir la porte à la possibilité d'autorisations ou de cessions implicites, contrairement au principe 
d'une cession explicite qui prévaut en droit d'auteur. En réalité, la solution semble avoir été motivée par des circonstances particulières, la Cour relevant que les photographies figuraient sur le site Web uniquement en basse définition et étaient protégées par un système anti-piratage rendant impossible leur exploitation par des tiers, conduisant à assimiler leur reproduction sur le site de l'agence à un moyen de proposer ces photographies à la vente conformément au mandat de l'agence.

\section{De nouvelles questions posées par la numérisation des fonds}

L'arrêt de la Cour de cassation de 2012 et la jurisprudence ultérieure en ce domaine illustre les difficultés d'application de la distinction entre droits sur un support photographique tangible et droits d'auteur sur l'image, lorsqu'existent de manière concurrente pour un même cliché différents tirages analogiques et des numérisations de plus ou moins bonne qualité.

Une décision du tribunal de grande instance de Paris de $2015^{7}$ s'est prononcée sur la propriété corporelle des photographies d'un photographe et des fichiers numérisés de ses photographies par son agence. Le litige opposait l'agence photographique Gamma-Rapho, qui avait fait faillite, et une de ses anciennes photographes, sur la propriété et la restitution de photographies. L'agence considérait qu'elle avait elle-même financé les copies numériques des photographies et s'opposait à l'exploitation de ces fichiers par la photographe sur son site personnel. Se posaient des questions complexes au regard des actes de cession du fonds de commerce intervenus et de la séparation à établir entre propriété sur des supports et droits immatériels. Le tribunal a recouru à la distinction traditionnelle entre l'œuvre et son support et a retenu dans ce contexte qu'un fichier numérique peut être considéré comme un support et "constitutif d'un bien meuble corporel».

Le tribunal a considéré que bien qu'elle fût titulaire des droits sur ses images, la photographe n'avait aucun droit sur les fichiers numérisés réalisés par l'agence GammaRapho $^{8}$. Dans son jugement du 13 mars 2015, le tribunal de grande instance de Paris a cependant limité l'indemnisation de l'agence à 1000 euros, dans la mesure où ces fichiers n'ont pas de valeur marchande, l'agence n'ayant pas l'autorisation de l'auteur de les exploiter. Cette décision a été très commentée dans le secteur de la photographie. Elle a aussi abouti à un résultat qui peut surprendre : une agence peut numériser les photos dont elle est en possession sans pouvoir les exploiter, tandis que la photographe qui détient les droits d'auteur sur ses clichés ne peut utiliser les numérisations de ces derniers réalisées par l'agence. Plus largement, elle témoigne de la difficulté d'appréhender juridiquement le fichier numérique en tant que bien meuble, catégorie traditionnelle du Code civil pensée avant tout en référence aux objets tangibles.

Par sa décision du 19 février $2016^{9}$, la cour d'appel de Paris a confirmé le jugement de première instance en considérant que les sept cent soixante-dix fichiers de numérisations de photographies de la photographe ne lui appartenaient pas, et a donné raison à l'agence de presse Gamma-Rapho qui en revendiquait la propriété. Le raisonnement conduit par la cour d'appel pour distinguer les objets en présence, photographies de départ et numérisations effectuées par l'agence, est éclairant :

Considérant que, si l'œuvre du photographe figure sur les fichiers numériques, ceux-ci sont composés du scan en haute définition des photographies argentiques mais également de légendes, d'éditing, de commentaires et d'informations textuelles réparties sur plusieurs champs dénommés IPTC qui ne peuvent plus être 
dissociés des photographies ; que la réalisation de ces fichiers exige plusieurs étapes dont la sélection de la photographie par des spécialistes, la réalisation d'un scan avec un matériel informatique spécifique, le renseignement des champs IPTC numériques constitués de données qui s'inscrivent dans le fichier image et qui apportent des informations sur le contexte de l'image grâce à un logiciel ; que ces opérations qui ont permis de réaliser la numérisation des photographies argentiques, propriété de Mme de $\mathrm{Y}$, sont dès lors le fruit de l'industrie et des investissements des sociétés Eyedea Presse et Hachette; qu'ainsi, la photographie argentique et le fichier numérique de la même image coexistent, le second ne conduisant pas à intégrer ou à supprimer l'existence ou la matière de la première.

La cour a dès lors estimé que la photographe, en diffusant sur son site des fichiers de haute définition, qui reprenaient les légendes et les numéros internes d'indexation de l'agence, alors qu'elle ne pouvait ignorer qu'elle ne détenait aucun droit sur ces fichiers et que l'accord de l'agence était requis, avait commis une faute.

\section{Le financement des supports et la preuve de la propriété}

Les tensions qui surviennent dans le secteur du photojournalisme se cristallisent fréquemment autour de la preuve de la propriété des supports des clichés, des tirages, et sur le fait de savoir qui a financé leur réalisation.

Dans un arrêt du 28 octobre $2015^{10}$, la Cour de cassation a jugé qu'un groupe de presse qui a financé les supports vierges et les frais techniques de développement de photographies est le propriétaire originaire des supports matériels des photographies. Il s'agissait en l'espèce d'un photographe ayant réalisé entre 1974 et 1984 des reportages pour un magazine et qui reprochait à son éditeur de ne pas lui avoir restitué les clichés photographiques dont il lui avait remis les négatifs aux fins de reproduction dans ce magazine, sans toutefois lui en avoir cédé la propriété corporelle. Pour s'opposer à cette demande, la société a soutenu être propriétaire des supports matériels des photographies litigieuses.

La cour d'appel, retenant que la société ne rapportait pas la preuve de l'acquisition des supports, avait condamné la société à payer des dommages-intérêts en réparation du préjudice patrimonial résultant de la non-restitution des clichés photographiques. La Cour de cassation, sous le visa de l'article 544 du code civil ${ }^{11}$ relatif à la propriété corporelle, et de l'article L. 111-3 du code de la propriété intellectuelle ${ }^{12}$, qui pose la distinction entre propriété corporelle et propriété immatérielle, a censuré l'arrêt des juges du fond dans sa décision du 28 octobre 2015 en énonçant la solution indiquée précédemment. Ce faisant, elle retient que l'éditeur est le propriétaire originaire des supports du seul fait de leur financement, sans qu'il soit besoin de rechercher une autre preuve, une cession par exemple.

Pour des services d'archives détenteurs de fonds photographiques d'origine privée, il est délicat d'identifier les transmissions successives de propriété sur les différents supports matériels, a fortiori s'il existe plusieurs tirages. Un journal dépositaire de photographies d'un photographe ne peut théoriquement pas les céder ni les déposer à son tour puisqu'elles ne lui appartiennent pas. Pour autant, des fonds à l'origine incertaine font partie de fait des collections publiques aujourd'hui. Il est important dès lors de revenir sur les différentes manières dont les archives peuvent détenir ou être propriétaires de fonds et de voir quels points de vigilance il est possible de préconiser. 


\section{La propriété des fonds photographiques entrés dans les collections publiques}

Il s'agit ici de passer en revue les différentes formes d'entrées de fonds photographiques dans les collections, selon qu'elles ont lieu à titre onéreux ou gratuitement, et les conséquences qu'il faut en tirer pour la propriété des supports photographiques.

Juridiquement, les fonds photographiques reçus par les archives de photographes ou de journaux sont des archives privées ${ }^{13}$. Or, l'article L. 212-16 du code du patrimoine ${ }^{14}$ précise que le classement de documents privés comme archives historiques n'emporte pas transfert à l'État de la propriété des documents classés. Il faut, pour que ce soit le cas, qu'intervienne une opération entraînant un transfert de propriété. Le $3^{\circ}$ de l'article L. 2112-1 du code général de la propriété des personnes publiques ${ }^{15}$ précise ainsi que font partie du domaine public mobilier d'une personne publique « les archives issues de fonds privés entrées dans les collections publiques par acquisition à titre onéreux, don, dation ou legs ".

Ces formes d'entrées dans les collections énumérées par la loi emportent un transfert de propriété au bénéfice de la collectivité locale dont dépend le service d'archives, ce qui rend les documents concernés inaliénables et imprescriptibles. Les différentes formes d'entrées de fonds photographiques dans les collections peuvent être distinguées selon qu'elles ont lieu à titre onéreux ou gratuitement - on parle alors de libéralités ${ }^{16}$.

\section{Les acquisitions onéreuses}

La première forme d'acquisition onéreuse est naturellement l'achat. Un service d'archives départemental peut acheter directement auprès de particuliers ou de professionnels des documents d'origine privée présentant un intérêt historique pour le département. L'État peut par ailleurs exercer un droit de préemption qui le subroge à l'adjudicataire ou au dernier enchérisseur au prix de la dernière enchère (Code du patrimoine, livre II, L. 212-32 à 212-36). En novembre 2012, l'État a ainsi racheté pour le compte de la Ville de Paris le fonds des archives photographiques de France-soir couvrant la période de 1980 à 2000, dans le cadre de la liquidation du journal.

En deuxième lieu, le mécanisme de la dation permet à un particulier de s'acquitter de certains impôts en nature (droits de mutation à titre gratuit et droit de partage) par la remise d'archives à l'État ${ }^{17}$. C'est une procédure exceptionnelle qui est subordonnée à un agrément délivré par le ministre chargé du budget après avis d'une commission nationale d'agrément. C'est grâce à ce dispositif que sont entrés dans les collections du musée national d'Art moderne des fonds photographiques de Man Ray, Marc Riboud, Brassaï et Henri Cartier-Bresson.

\section{Les libéralités}

Le legs, d'abord, consiste pour une personne à donner une partie de ses biens après son décès par dispositions testamentaires. Il porte en général dérogation aux règles légales de la dévolution successorale, l'objectif de l'auteur d'un testament consistant, soit à attribuer à un de ses héritiers légaux une part d'un montant excédant la part d'héritage que la loi lui réserve, soit à attribuer tout ou partie de sa succession à une personne qui 
n'y était pas normalement appelée, par exemple un service d'archives à qui seront remis des documents ${ }^{18}$.

Un legs est accepté par écrit par le légataire, et implique un transfert de propriété. Selon les cas, le legs sera accepté par la direction des Archives de France, le conseil départemental ou le conseil municipal. Le photographe Marc Riboud, décédé en 2016, avait par exemple prévu un legs par un accord passé en 2015 avec le musée national des Arts asiatiques - Guimet ${ }^{19}$.

À la différence du legs, la donation implique que le transfert de propriété du donateur au service d'archives intervienne du vivant du donateur. L'article $931 \mathrm{du}$ Code civil ${ }^{20}$ exige qu'elle ait lieu par acte notarié, sous peine de nullité. En pratique toutefois, la jurisprudence admet le don «manuel» pour des documents n'ayant aucune valeur marchande, tandis qu'on réserve la donation notariée pour des fonds prestigieux et de valeur. Tel a été le cas lorsque Willy Ronis a fait don, par deux donations de 1983 et 1989, complétées par son testament en 2009, de l'intégralité de son œuvre à l'État ${ }^{21}$. Il est essentiel pour le service d'archives donataire que le don soit accompagné d'une lettre d'intention du donateur, et de garder trace de cette lettre d'intention. Le portail Web des Archives de France propose des modèles de lettres d'intention de donation et d'acceptation de donation ${ }^{22}$.

Il est important par ailleurs d'établir une procédure à suivre, en s'assurant en premier lieu que le donateur est bien le propriétaire des archives en question et en respectant plusieurs étapes : lettre d'intention de don, lettre d'acceptation, remise matérielle avec reçu, lettre de remerciement précisant le numéro d'inscription au registre des entrées par voie extraordinaire.

\section{Le cas du dépôt}

Juridiquement, détenir un objet n'implique pas toujours que l'on en soit propriétaire. Tel est le cas du dépôt, que l'article 1915 du Code civil définit comme « un acte par lequel on reçoit la chose d'autrui, à la charge de la garder et de la restituer en nature ». Un dépôt est révocable à tout moment et peut être dénoncé par l'une ou l'autre des parties. Au décès du déposant, la propriété des archives passe à ses héritiers, qui peuvent révoquer le dépôt ou le proroger. Ainsi, lorsqu'un service d'archives a reçu des archives de presse en dépôt, le propriétaire initial conserve ici son droit de propriété mais confie ses photographies à un service d'archives, dépositaire, à des fins de conservation et de communication.

Les Archives diplomatiques du Quai d'Orsay, par exemple, se sont considérablement enrichies grâce au dépôt d'archives privées: en 1950, de la collection Jules Herbette, diplomate en poste à Berlin entre 1886 et 1896, ou en 1968, de la collection Édouard de La Selle, diplomate en poste à Rome et à Vienne de 1860 à $1865^{23}$.

Les dépôts sont réalisés par acte sous seing privé, c'est-à-dire par un contrat de dépôt assorti de conditions et de clauses de garantie pour le service public. L'important est ici de formaliser la relation unissant le déposant, qu'il s'agisse d'un photographe ou d'un groupe de presse, et le service d'archives. Le portail Web des Archives de France propose une formule-type de contrat de dépôt d'archives privées ${ }^{24}$. 


\section{La prescription acquisitive}

Il arrive que des services d'archives aient en leur possession des fonds photographiques sans pour autant connaître les modal ités d'entrée de ces fonds dans leurs collections, en l'absence de document précisant l'intention du producteur ou de contrat valant transfert de propriété. Si cette méconnaissance peut sembler particulièrement rédhibitoire pour la valorisation d'un fonds, une solution consiste à recourir à un mécanisme prévu par le Code civil, la prescription acquisitive.

En droit français, un fonds d'archives est considéré comme un bien meuble et obéit au régime du droit commun de la propriété organisé par le Code civil. L'article 2276 alinéa 1 er du Code civil ${ }^{25}$ prévoit qu' " en fait de meubles, la possession vaut titre », ce qui a une double conséquence, en matière d'acquisition et de preuve. Cette règle dite de la prescription acquisitive permet au possesseur de bonne foi d'un bien meuble, d'une part d'en acquérir immédiatement la propriété, et d'autre part, d'être présumé propriétaire de ce bien jusqu'à preuve du contraire.

Pour que la prescription acquisitive ait lieu, il est nécessaire de réunir cinq conditions cumulatives, en application de l'article 2261 du Code civil ${ }^{26}$ : la possession doit être continue et non interrompue, paisible, publique, non équivoque, et exercée à titre de propriétaire ${ }^{27}$. Lorsqu'on est en présence de l'ensemble de ces conditions, le possesseur du bien meuble est présumé propriétaire dès qu'il a le bien entre ses mains jusqu'à preuve du contraire, et le véritable propriétaire ne peut plus revendiquer son bien auprès du possesseur de bonne foi. La Cour de cassation considère que les conditions de la possession doivent être appréciées au moment de l'entrée en possession ${ }^{28}$.

Un propriétaire lésé ne se trouve pas pour autant démuni. Il peut exercer plusieurs actions soumises à des délais différents, selon qu'il s'est dessaisi de son bien volontairement ou involontairement. Dans le premier cas, il peut revendiquer son bien, dans un délai de cinq ans « à compter du jour où le titulaire d'un droit a connu ou aurait dû connaitre les faits lui permettant de l'exercer ", en application de l'article 2224 du Code civil ${ }^{29}$ qui fixe le délai de droit commun des actions mobilières. Par exemple, en présence d'un contrat de dépôt, le propriétaire peut agir contre le détenteur qui a reçu le meuble du propriétaire à titre précaire dans ce délai de cinq ans : il s'agit alors d'une action personnelle en restitution, née du contrat.

S'agissant du propriétaire dépossédé de son bien contre son gré à la suite d'une perte ou d'un vol, il peut le revendiquer à condition d'agir dans les trois ans à compter du jour de la perte ou du vol, en application de l'article 2276 alinéa 2 du Code civil ${ }^{30}$. Tel peut être le cas contre un acquéreur ayant acquis de bonne foi le meuble perdu ou volé, par exemple dans un marché, une foire ou une vente publique. L'action en revendication contre un acquéreur ayant obtenu de mauvaise foi le meuble perdu ou volé se prescrit en revanche selon un délai de cinq ans.

\section{L'application de la prescription acquisitive aux archives}

Interrogeons-nous à partir de la situation concrète suivante: un service d'archives qui possède un fonds photographique et s'en considère propriétaire à l'appui d'un contrat de vente ou d'une donation se trouve confronté à la revendication d'un tiers, qui avance que 
le service d'archives n'est en réalité que le détenteur précaire du bien en vertu d'un contrat de dépôt.

La réponse de la jurisprudence est constante sur ce point : il existe une présomption de propriété sur le meuble du possesseur et la charge de la preuve pèse sur celui qui le revendique. S'il ne peut y parvenir, le possesseur du meuble sera donc considéré comme son véritable propriétaire. Le tiers revendiquant peut renverser la présomption en invoquant plusieurs éléments, comme la précarité du titre du possesseur : le propriétaire revendiquant produit par exemple un contrat de dépôt démontrant la détention précaire $\mathrm{du}$ possesseur.

Les services d'archives peuvent ainsi recourir, en l'absence d'écrit constatant un don, par exemple, à la règle de la prescription acquisitive, s'ils peuvent démontrer que leur possession répond aux exigences légales mentionnées précédemment.

Sur la base des informations disponibles, il faut alors déterminer une date d'entrée du fonds d'archives. Cette date servira au calcul de la période de prescription acquisitive de cinq ans. À l'expiration du délai, on peut considérer que le service d'archives en a acquis la propriété. À titre d'exemple, à partir des règles du Code civil du Québec - qui sont très proches du mécanisme français - les Bibliothèque et Archives nationales du Québec proposent une procédure d'application de la prescription acquisitive ${ }^{31}$.

Pour l'avenir, on soulignera l'importance pour les services d'archives de garder toute pièce, tout contrat ou autre écrit permettant d'attester de la propriété des fonds qu'ils conservent afin d'être en mesure d'établir si l'on est en présence d'une cession ou d'un dépôt. L'importance d'un écrit se justifie également du fait qu'en vertu de l'article $\mathrm{L}$. 213-6 du Code du patrimoine ${ }^{32}$, la communicabilité des fonds d'archives privées reçus à titre de don, de legs, de cession, de dépôt ou de dation peut être définie par les propriétaires et doit alors être respectée. Si elle ne l'a pas été, la communication des documents respectera la réglementation sur les archives en vigueur.

\section{NOTES}

1. - Voir dans ce numéro : MESLEM, Angelina, EON, Wilfrid et VIALAR, Clémentine. « Le fonds Jean Lattes : une archive photographique en construction ", In Situ [En ligne], 36|2018, mis en ligne le 15 octobre 2018, consulté le 18 octobre 2018. URL: http://journals.openedition.org/ insitu/17738. Voir également le site: https://archives.yvelines.fr/article.php? larub=203\&titre=nos-collections [consulté le 23/08/2018].

2. - Voir les sites : http://archives.cantal.fr/?id=recherche_grandpublic_detail\&doc=accounts\% 2Fmnesys_ad15\%2Fdatas\%2Fir\%2FS\%C3\%A9ries\%20th\%C3\%A9matiques\%2FDocuments\%20figur\% C3\%A9s\%2FFRAD015_31_NUM.xml et http://www.archives.cg19.fr/recherche/serie/id/1238 [consultés le 23/08/2018].

3. - Voir sur le site : Cour de Cassation, Chambre civile 1, du 20 décembre 1966, publié au bulletin [consulté le 23/08/2018].

4. - Les agences sont définies à l'article $1^{\mathrm{er}}$ de l'ordonnance $\mathrm{n}^{\circ} 45-2646 \mathrm{du} 2$ novembre 1945 portant réglementation des agences de presse : «Sont considérées comme agences de presse, au 
sens de la présente ordonnance, les entreprises commerciales qui collectent, traitent, mettent en forme et fournissent à titre professionnel tous éléments d'information ayant fait l'objet sous leur propre responsabilité d'un traitement journalistique et dont la moitié au moins du chiffre d'affaires provient de la fourniture de ces éléments à des entreprises éditrices de publications de presse, au sens de la loi $\mathrm{n}^{\circ}$ 86-897 du $1^{\mathrm{er}}$ août 1986 portant réforme du régime juridique de la presse, à des éditeurs de services de communication au public par voie électronique et à des agences de presse.» L'Association pour la gestion de la sécurité sociale des auteurs (AGESSA) considère comme agences de presse photographique celles dont la moitié du chiffre d'affaires provient de la vente d'œuvres photographiques.

5. - Voir sur le site: Tribunal de grande instance de Paris, Chambre civile 3, 21 décembre 2007, 07/02017 [consulté le 23/08/2018].

6. - Voir sur le site : Cour de cassation, Première chambre civile, arrêt $n^{\circ} 612$ du 30 mai 2012 (10-17.780) [consulté le 23/08/2018].

7. - Tribunal de grande instance de Paris, $3^{\mathrm{e}}$ chambre, $3^{\mathrm{e}}$ section, jugement du 13 mars 2015, Gamma-Rapho c/Mme N-O de Y.

8. - Extrait du jugement: «La société Gamma-Rapho se trouve donc propriétaire des fichiers numérisés des photographies de M.-L. de Decker, biens meubles corporels qui lui ont été transmis, sans pour autant pouvoir les exploiter, en l'absence d'autorisation sur ce point par la photographe. L'utilisation par la défenderesse sur son mur facebook et sur son site Internet de fichiers numérisés, [...] sans l'autorisation de celle-ci est fautive ».

9. - Cour d'appel de Paris, pôle 5 - chambre 2, arrêt du 19 février 2016, Gamma-Rapho c/Mme N.O. de Y.

10. - Voir sur le site : Cour de cassation, civile, Chambre civile 1, 28 octobre 2015, 14-22.207, publié au bulletin [consulté le 23/08/2018].

11. - Voir l'article 544 du code civil « La propriété est le droit de jouir et disposer des choses de la manière la plus absolue, pourvu qu'on n'en fasse pas un usage prohibé par les lois ou par les règlements ».

12. - Voir l'article L. 111-3 du code de la propriété intellectuelle : "La propriété incorporelle définie par l'article L. 111-1 est indépendante de la propriété de l'objet matériel. L'acquéreur de cet objet n'est investi, du fait de cette acquisition, d'aucun des droits prévus par le présent code, sauf dans les cas prévus par les dispositions des deuxième et troisième alinéas de l'article L. 123-4. Ces droits subsistent en la personne de l'auteur ou de ses ayants droit qui, pourtant, ne pourront exiger du propriétaire de l'objet matériel la mise à leur disposition de cet objet pour l'exercice desdits droits. Néanmoins, en cas d'abus notoire du propriétaire empêchant l'exercice du droit de divulgation, le tribunal de grande instance peut prendre toute mesure appropriée, conformément aux dispositions de l'article L. 121-3 ».

13. - L'article L. 211-5 du Code du patrimoine définit les archives privées en creux comme étant l'ensemble des documents qui ne sont pas des archives publiques, c'est-à-dire qui n'entrent pas dans la définition précédente de l'article L. 211-4. La notion d' « archives privées » recouvre ainsi des réalités très variées telles que des archives personnelles et familiales, des archives d'entreprise ou des archives religieuses qui peuvent être réparties selon l'identité du producteur. 14. - Voir L'article L. 212-16 du code du patrimoine.

15. - Voir l'article L. 2112-1 du code général de la propriété des personnes publiques.

16. - Pour une vision complète de ces enjeux, voir : NOUGARET, Christine et ÉVEN, Pascal (dir.). Les Archives privées. Manuel pratique et juridique. Paris : la Documentation française, 2008.

17. - Voir sur le site : Article 1716 bis du code général des impôts [consulté le 23/08/2018].

18. - Le legs est encadré par les articles 1014 et suivants du Code civil : voir sur le site https:// www.legifrance.gouv.fr/affichCode.do?

idSectionTA=LEGISCTA000006150551\&cidTexte=LEGITEXT000006070721 [consulté le 23/08/2018].

19. - Voir le site : http://www.guimet.fr/communiques-de-presse/. 
20. - Voir L'article 931 du Code civil.

21. - Voir le site : http://www.culturecommunication.gouv.fr/Actualites/Willy-Ronis-un-fondsphotographique-d-une-richesse-exceptionnelle [consulté le 23/08/2018].

22. - Voir le site : https://francearchives.fr/article/37730 [consulté le 07/09/2018].

23. - Voir le site : http://www.diplomatie.gouv.fr/fr/archives-diplomatiques/s-orienter-dans-lesfonds-et-collections/images/article/origine-et-presentation-des-fonds [consulté le 23/08/2018].

24. - Voir le site : https://francearchives.fr/article/38061 [consulté le 23/08/2018].

25. - Voir L'article 2276 alinéa 1er du Code civil.

26. - Voir l'article 2261 du Code civil.

27. - Un prêt, par exemple, oblige à une restitution ultérieure de l'objet.

28. - Cass., Civ. $1^{\text {re }}, 4$ janvier 1972.

29. - Voir l'article 2224 du Code civil.

30. - Voir l'article 2276 alinéa 2 du Code civil.

31. Voir le site: http://www.banq.qc.ca/documents/archives/archivistique_gestion/ partenaires/serv_archives_privees_agrees/Procedure_prescription_acquisitive\%282016-10-19\% 29.pdf [consulté le 23/08/2018].

32. - Voir l'article L. 213-6 du Code du patrimoine.

\section{RÉSUMÉS}

De nombreux services d'archives conservent des fonds photographiques de presse importants, composés de photographies de provenances diverses et aux objets non authentifiés, ce qui rend délicate l'identification de la propriété matérielle des différents éléments composant ces fonds. Cette difficulté résulte notamment de la diversité des acteurs au sein du secteur du photojournalisme et du fait que les pratiques liées aux supports matériels des photographies ne sont pas harmonisées. La transformation numérique de l'économie transforme à la fois les relations entre intervenants traditionnels dans la chaîne de création et le nombre d'images accessibles au grand public. Des conflits sont apparus entre des photographes et des agences de presse, qui portent la plupart du temps sur la restitution aux photographes de leurs originaux ou l'accès à ces derniers lorsque les photographes n'ont pas conservé de copies de ces derniers en amont. Dans ce contexte, la jurisprudence relative aux pratiques encadrant le transfert de propriété des supports photographiques dans les relations entre photographes et agences de presse apporte un éclairage intéressant pour les archives, qui interviennent à l'autre bout de la chaîne. En second lieu, il convient de s'interroger sur les différentes modalités d'entrée des archives de presse dans les collections publiques et leur impact sur la propriété matérielle. Sont examinées à ce titre les différentes formes d'entrées de fonds photographiques dans les collections, selon qu'elles ont lieu à titre onéreux ou gratuitement, et les conséquences qu'il faut en tirer pour la propriété des supports photographiques. À ce titre est abordée la possibilité pour des services d'archives d'appliquer la règle de la prescription acquisitive aux fonds photographiques privés dont les modalités d'entrée dans les collections sont inconnues, en l'absence de document précisant l'intention du producteur du fonds.

There are many archive services which today hold significant collections of press photos. These may come from a variety of different origins-often unidentified ones-and this makes it difficult to identify the material property of the elements which make up the collections. Photojournalism 
has always involved a broad variety of actors, and practices where the material medium of the photograph is concerned are by no means standardised. Digitisation is now changing the relations between the traditional actors in the processes of picture creation and is also increasing the number of pictures available to the general public. The conflicts which have arisen between photographers and agencies often concern the restitution to the photographers of their originals or the photographers' access to these originals when copies of them were not kept. In this context, the jurisprudence relating to practices in the transfer between photographers and press agencies of ownership of photographic works may throw some useful light on the archival questions which arise at the end of the process. It is also necessary to examine how the photo collections entered public archives depots, since these modalities of accession may influence questions of ownership. In this article, the different ways a photo collection may enter a public archive depot are examined. Depending on whether the collection was purchased or donated, the consequences are not the same where the ownership of the originals is concerned. The possibility for archival services to apply the principle of prescriptive acquisition to the private photo collections they hold is examined, in cases where the origins of the collections are not clearly determined and where there is no document to stipulate the intentions of the person or service that produced the collection.

\section{INDEX}

Keywords : copyright, legal, property, material, photography, press, archives, medium, print, digitisation, proof of ownership, acquisitive property

Mots-clés : droit, juridique, propriété, matérielle, photographie, presse, archives, support, tirage, numérisation, preuve de la propriété, propriété acquisitive

\section{AUTEUR}

\section{SAMUEL BONNAUD-LE ROUX}

Chargé de mission au bureau de la propriété intellectuelle, Secrétariat général, ministère de la Culture samuel.bonnaud-le-roux@culture.gouv.fr 